\title{
Failure Modes of Doubly Supported Capacitive RF MEMS Switches
}

\author{
Li-Xian Zhang ${ }^{1.2}$, Jin-Wen Zhang ${ }^{2}$, Ya-Pu Zhao ${ }^{1 *}$, Tong-Xi Yu ${ }^{3}$
}

1. State Key Laboratory of Nonlinear Mechanics (LNM), Institute of Mechanics, Chinese Academy of Sciences, Beijing 100080, China

2. Institution of Microelectronics, Peking University, Beijing 100871,China

3. Department of Mechanical Engineering, Hong Kong University of Science and Technology, Clear Water Bay, Kowloon, Hong Kong SAR, China

\begin{abstract}
For the design of radio frequency micro-electro-mechanical systems (RF MEMS) switches, the reliability issue becomes increasingly important. This paper represents some failure phenomena of doubly supported capacitive RF MEMS switches that include observable destruction failure and directly measurable parameter degradation obtained from the actuating-voltage testing and scanning electron microscope (SEM) observation. The relevant failure modes as well as their failure mechanisms are identified.
\end{abstract}

Keywords: RF MEMS, reliability, failure mode, fracture, stiction, residual stress, stress gradient

\section{Introduction}

In recent years, radio frequency micro-electromechanical systems (RF MEMS) emerged and have developed rapidly around the world $[1,2]$. Compared with semiconductor switches such as p-i-n (PIN) diodes and field-effect transistor (FET) switches widely used in microwave and monolithic microwave integrated circuits (MMICs), RF MEMS switches offer higher performance such as low insertion loss, good isolation, low return loss, high frequency, good Q-factor, low cost and power consumption.

A doubly supported capacitive RF MEMS switch usually consists of two parallel plates. One plate is fixed on the substrate and the other is formed by $\mathrm{Au}$ or $\mathrm{Cu}$ thin film prepared by electroplating process; the latter called the "bridge" is suspended over the transmission line. A bias voltage is applied and varies as the gap between them changes. Schematic of a simple structure and SEM of the switches are presented in Figs. 1 and 2, respectively. For the design, various structural geometries of the hinges and movable plate are investigated to obtain low insertion loss and low actuation voltage.

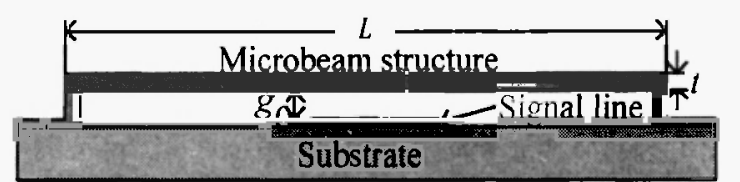

(a)

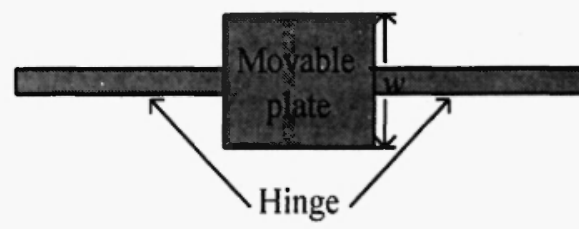

(b)

Fig. 1. Schematic of doubly supported capacitive RF MEMS switches (a) Side view (b) Top view

\footnotetext{
* To whom all correspondence should be addressed. E-mail: yzhao@lam.imechac.cn (Ya-Pu Zhao).
} 


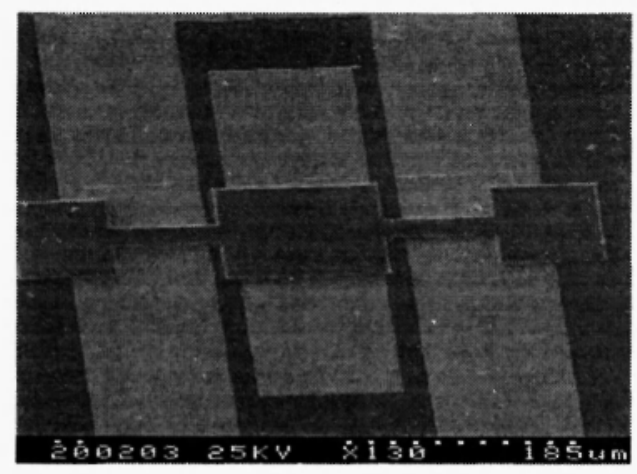

Fig. 2. SEM of a clamped capacitive RF MEMS switch

A failure is said to have occurred when a device no longer performs the required functions under the stated conditions within the stated period of time [3]. The failure analysis is inevitable and significant to the design and the microfabrication of capacitive RF MEMS switches. In this paper, we carry out an investigation about failure modes based on the phenomena observed in the actuating-voltage testing and scanning electron microscope (SEM) observation.

\section{Experiment and Failure Modes}

The actuating-voltage testing is carried out using Keithley Model 82-WIN Synchronous C-V Measurer in order to obtain the threshold voltage. The theoretical value of threshold voltage can be calculated by [4]

$$
V_{m}=\sqrt{\frac{256}{27} \frac{\widetilde{E} g_{0}{ }^{3} t^{3}}{\varepsilon_{0} L^{4}}},
$$

where $\widetilde{E}$ denotes the effective modulus, $\varepsilon_{0}$ is the permittivity of air, $g_{0}$ is the gap between the movable plate and the signal line, $t$ and $L$ denote the thickness and length of the movable plate, respectively. The relevant values of these parameters are listed in Table 1.

The applied voltage is limited to 40 volts because the value calculated from Eq. (1) is about 25 volts. The failure phenomena observed during the testing and SEM observation can be catalogued into three types; i.e., fracture, stiction and residual stress induced deformation modes. The former two modes belong to irreversible category that involves the destruction of device, rendering it inoperable. The latter two modes belong to degradation failure that causes the device parameters to operate far beyond the normal range of operation. A typical and natural $\mathrm{C}-\mathrm{V}$ diagram of the capacitance RF MEMS switches is shown in Fig. 3.

Table 1. The parameters of the capacitive RF MEMS switches concerned in this paper

\begin{tabular}{cll}
\hline Variables & \multicolumn{1}{c}{ Parameters } & \multicolumn{1}{c}{ Value } \\
\hline$\tilde{E}$ & effective modulus & $\sim 1.65^{*} 10^{11} \mathrm{~N} / \mathrm{m}^{2}$ \\
$g_{0}$ & original gap & $1.3-1.8 \mu \mathrm{m}$ \\
$L$ & Length & $260-400 \mu \mathrm{m}$ \\
$t$ & Thickness & $2-4 \mu \mathrm{m}$ \\
$w$ & Width & $120 \mu \mathrm{m}$ \\
\hline
\end{tabular}

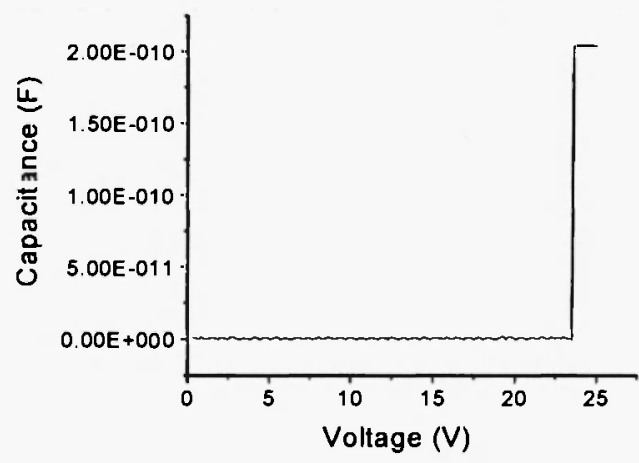

Fig. 3. C-V diagram of natural device whose parameters operate inside the normal range

\subsection{Fracture}

Fracture occurs when the maximum stress in the device is greater than the stress tolerance of the material during the normal operation or the fabrication process. In principle, this failure mode is relatively easy to be observed and predicted. A SEM photo for fracture is shown in Fig. 4.

To avoid the fracture, it is required to design the device with the applied stress below the material strength. However, the mechanical properties of materials required for the design are often unavailable at the microscopic level. In addition, residual stress inhering in the 
micromachining structure and various geometries of the device are required to be taken into consideration, and fatigue arising from repeated operation is often concerned.

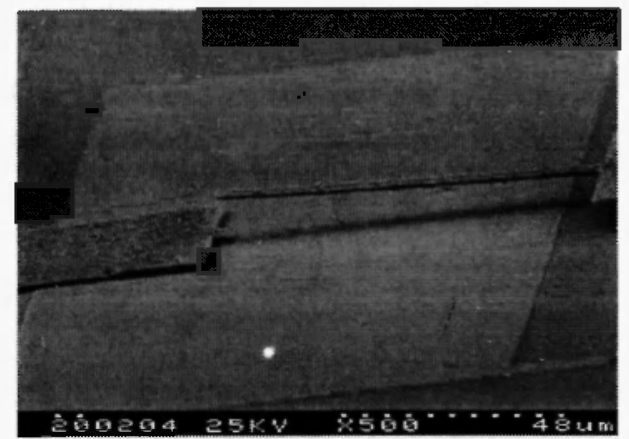

Fig. 4. SEM of fracture mode

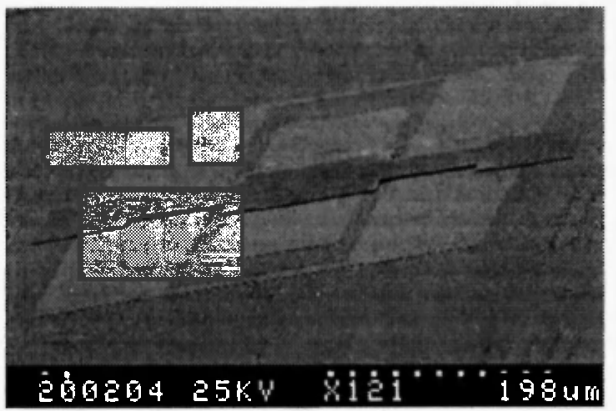

(a)

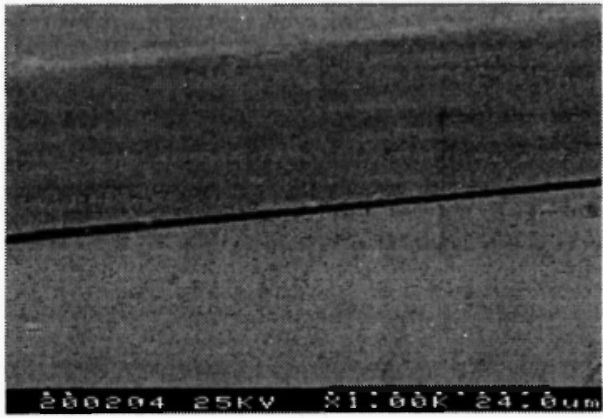

(b)

Fig. 5. SEM of stiction mode (a) Total switch (b) Adhesion of the switch to the substrate

\section{2, Stiction}

Due to the high ratio of surface area to volume in the microbeam structure, it is specifically prone to adhere to the substrate when it is bent by an applied force or inherent stress. In the present study, this failure mode has two types. One is that the movable plate snaps onto the lower plate subject to an applied voltage but still adheres when the applied voltage is removed. Another is the adhesion between the movable and the lower plates before applying voltage. The relevant SEM photos are shown in Fig. 5. Fig. 5 (a) presents the total device, and Fig. 5 (b) represents middle part of the device that adheres to the substrate.

Permanent adhesion occurs owing to the large interfacial forces compared with the restoring force of the deflected structure. Van der Waals force, electrostatic charging and the effect of surface roughness all participate in the surface stiction mechanism. Further analysis can be referring to other literatures $[5,6]$.

\subsection{Residual stress induced deformation}

This failure mode occurs mainly due to high residual stress and the residual stress gradient inside the microstructures. The actual electromechanical behaviors of this mode vary with the different structure geometry of RF MEMS switches.

For a straight structure, the device becomes convex as a result of large compressive residual stress, rendering it not actuated even if under 40 volts. The typical C-V curve shown in Fig. 6 with fluctuation is due to the environment noise ranging between $-0.9 \mathrm{pf}$ and $1.5 \mathrm{pf}$. One SEM photo of the device is shown in Fig. 7. The larger is the residual stress inhering in the device, the higher is the threshold voltage.

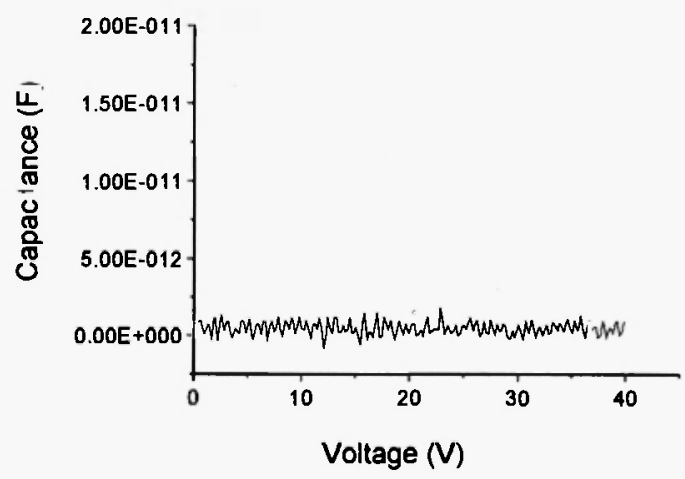

Fig. 6. C-V diagram of convex switch not actuated 


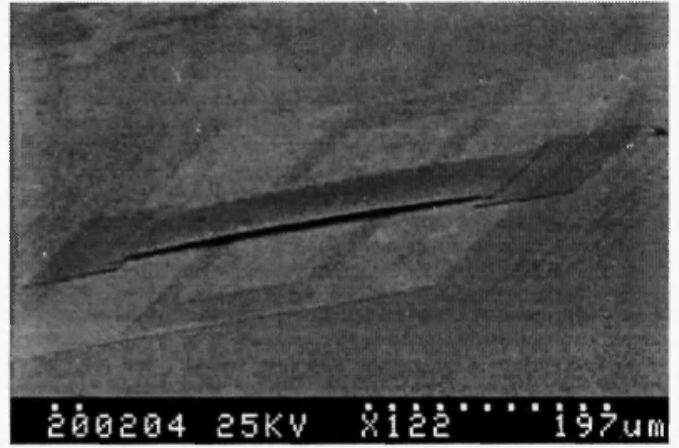

Fig. 7. SEM of one convex RF switch not actuated under 40 volts of applied voltage

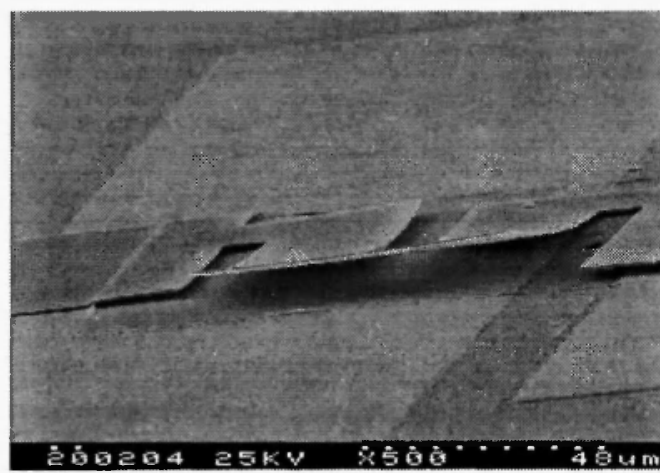

Fig. 8. SEM of torsion behavior with a zigzag structure

For a zigzag structure, evident torsion occurs due to high intrinsic stress, stress gradient and the incomplete symmetry of structure geometry as shown in Fig. 8, rendering its instability and threshold voltage boosted. On the other hand, the zigzag structure can increase its flexibility to reduce the actuating voltage.

The evident discrepancy between the theory and experiment on the parameter and appearance, is attributed to large residual stress and the stress gradient arising from the fabrication process. These factors can impose serious influence on both to straight and zigzag structures. Therefore the in-depth research of residual stress is of importance for the reliability design of capacitance RF MEMS switches.

\section{Conclusion}

The failure analysis of capacitive RF MEMS switches is significant to their design, fabrication and applications. Typical failure phenomena, obtained from actuating-voltage testing and SEM observation are presented. Three failure modes, i.e., fracture, stiction and residual stress induced deformation, are described in this paper. The associated failure mechanisms are also identified. Interfacial forces, stress gradient and structure geometry are responsible for those mechanisms. The qualitative analysis is beneficial to the reliability design of the device.

Further experiment, numerical simulation and quantitative analysis are currently in progress.

\section{Acknowledgments}

The supports from National Natural Science Foundation of China, Chinese Academy of Sciences and the Joint Laboratory of Microsystems between the Institute of Mechanics (CAS) and HKUST are gratefully acknowledged.

\section{References}

[1] Yao, J.J. Topical review: RF MEMS from a device perspective, J. Micromech. Microeng. 10(2000) R9-R38

[2] Feng, Z.P., Zhang, H.T. et al. MEMS-based variable capacitor for millimeter-wave applications, Proceedings of Solid-State Sensor and Actuator Workshop, Hilton Head Island, South Carolina, (2000) 255-258

[3] Man, K.F. MEMS reliability for space applications by elimination of potential failure modes through testing and analysis, 1999 SPIE Symposium on Micromachining and Microfabrication, Santa Clara, California, September 21-23, (1999)

[4] Osterberg, P.M. and Senturia, S.D. M-test: a test chip for MEMS material property measurement using electrostatically actuated test structures, J Microelectromech Syst. 6 (2) (1997) 107-118

[5] Zhao, Y.P. and Yu, T.X. Failure modes of MEMS and microscale adhesive contact theory, International Journal of Nonlinear Sciences and Numerical Simulation, 1(2000) 361-371

[6] Zhao, Y.P., Wang, L.S. and Yu, T.X. Mechanics of adhesion in MEMS - A review, Journal of Adhesion Science and Technology, Special Issue on Adhesion Aspects in MEMS, in press. 